\title{
Improved big bang nucleosynthesis constraints on heavy neutral leptons
}

\author{
A. Boyarsky, ${ }^{1}$ M. Ovchynnikov $\odot,{ }^{1}$ O. Ruchayskiy $\odot,{ }^{2}$ and V. Syvolap ${ }^{2}$ \\ ${ }^{1}$ Instituut-Lorentz for Theoretical Physics, Universiteit Leiden, \\ Niels Bohrweg 2, 2333 CA Leiden, The Netherlands \\ ${ }^{2}$ Niels Bohr Institute, University of Copenhagen, Blegdamsvej 17, DK-2100 Copenhagen, Denmark
}

(Received 17 August 2020; accepted 24 May 2021; published 16 July 2021)

\begin{abstract}
We constrain the lifetime of thermally produced heavy neutral leptons (HNLs) from big bang nucleosynthesis. We show that even a small fraction of mesons present in the primeval plasma leads to the overproduction of primordial helium-4. This constrains the lifetime of HNLs to be $\tau_{N}<0.02 \mathrm{~s}$ for masses above the mass of the pion (as compared to $0.1 \mathrm{~s}$ reported previously). In combination with accelerator searches, this allows us to put a new lower bound on the HNL masses and define the "bottom line" for HNL searches at the future Intensity Frontier experiments.
\end{abstract}

DOI: 10.1103/PhysRevD.104.023517

\section{INTRODUCTION}

Heavy neutral leptons (HNLs or right-handed neutrinos) are hypothetical particles capable of explaining neutrino masses and oscillations [1] and resolving other beyond-theStandard-Model phenomena, i.e., the origin of the baryon asymmetry of the Universe (see, e.g., Ref. [2]) and the nature of dark matter [3]. Extending the Standard Model (SM) with exactly three HNLs with masses below the electroweak scale and no other heavy physics allows to explain all three beyond-the-Standard-Model phenomena [4-6]. An attractive feature of the models with $\mathrm{MeV}-\mathrm{GeV}$-scale HNLs is that they can be tested by a combination of accelerator searches at the LHC (see, e.g., Refs. [1,7] and references therein) and future intensity frontier experiments (see, e.g., Ref. [8]).

The single requirement that HNLs contribute sizably to the masses of neutrinos combined with existing accelerator exclusions does not allow us to put a lower bound on the HNL mass. Such a lower bound can be obtained if we add to the picture constraints from big bang nucleosynthesis (BBN). Primordial abundances of ${ }^{4} \mathrm{He}$ and $\mathrm{D}$ are measured with high accuracy [9-14]. Their agreement with the Standard Model predictions [15-19] serves as one of the "pillars" of modern cosmology.

The success of the Standard-Model-based BBN (SBBN) predictions permits using it to constrain hypothetical particles with lifetimes as small as $\mathcal{O}\left(10^{-2}\right)$ s (see, e.g., Ref. [20] for a review). In particular, decays of HNLs in MeV-temperature plasma affect two observable quantities:

Published by the American Physical Society under the terms of the Creative Commons Attribution 4.0 International license. Further distribution of this work must maintain attribution to the author(s) and the published article's title, journal citation, and DOI. Funded by SCOAP ${ }^{3}$. (i) the abundances of light elements (in this paper we consider only ${ }^{4} \mathrm{He}$ ), and (ii) the effective number of relativistic species $N_{\text {eff. }}$. This was used to put an upper bound on the HNL lifetime [21-32]. These bounds were obtained using decays of HNLs into electromagnetic particles and neutrinos.

The goal of this paper is to derive BBN bounds for HNLs that can decay into mesons. The effect of mesons on the $\mathrm{MeV}$ plasma is qualitatively different as they interact with protons and neutrons via strong interactions. Although the lifetimes of mesons, $\tau_{\text {meson }} \sim 10^{-8} \mathrm{~s}$, are orders of magnitude smaller than any relevant BBN time scales, they can be present in the plasma as long as HNLs are still abundant and decay. $p \rightarrow n$ and $n \rightarrow p$ reactions driven by mesons have a cross section $\mathcal{O}\left(10^{16}\right)$ times larger than that of weak processes that are normally responsible for the $p \leftrightarrow n$ conversion in the absence of mesons (e.g., in Standard Model BBN). Moreover, these reactions, in both directions, have no threshold and roughly equal cross sections (due to isotopic symmetry).

Therefore, the presence of even a small fraction of mesons in the plasma quickly equilibrates the number densities of protons and neutrons. Once HNLs decay, mesons also disappear (instantaneously, as compared to the time scales relevant for $\mathrm{BBN}$ ), and the neutron-to-proton ratio $n_{n} / n_{p}$ relaxes solely due to the SM (weak) processes. If the HNL's lifetime is short enough, $n_{n} / n_{p}$ relaxes to its $\mathrm{SM}$ value before weak reactions freeze out, leaving no observable effect. However, if HNLs (and mesons) survive until $T \simeq 1.5 \mathrm{MeV}$ and below, there is not enough time to completely relax down to the SBBN value. This residual effect leads to a strong upper bound on the HNL lifetime.

In this work, we demonstrate for the first time that the meson-driven effect strengthens the upper bound on the HNL lifetime by a factor $\sim 5$ (down to $0.02 \mathrm{~s}$ ) as compared to the previous work [22]. In the context of dark scalars, the 
meson-driven $p \leftrightarrow n$ conversion and its influence on BBN were studied in Ref. [33-38].

For temperatures corresponding to such short lifetimes, all Standard Model particles are in thermal equilibrium. This makes all other effects of HNLs on BBN irrelevant and allows us to derive the bounds purely analytically, avoiding any computations of complicated Boltzmann equations.

\section{MESON-DRIVEN $\boldsymbol{p} \leftrightarrow \boldsymbol{n}$ CONVERSION}

Sufficiently heavy HNLs can decay into mesons $h=\pi$, $K$, etc. (see Refs. $[39,40]$ or Appendix C). Charged pions drive the $p \leftrightarrow n$ conversion via [33]

$$
\pi^{-}+p \rightarrow n+\pi^{0} / \gamma, \quad \pi^{+}+n \rightarrow p+\pi^{0} .
$$

The cross section of these reactions is very large:

$$
\frac{\left\langle\sigma_{p \leftrightarrow n}^{\pi} v\right\rangle}{\left\langle\sigma_{p \leftrightarrow n}^{\text {Weak }} v\right\rangle} \simeq \frac{1}{G_{F}^{2} m_{p}^{2} T^{2}} \sim 10^{16}\left(\frac{1 \mathrm{MeV}}{T}\right)^{2} .
$$

The large cross section, absence of a threshold, and isotopic symmetry of these processes mean that if pions are present in the plasma in amounts at least comparable to that of baryons, they drive the number densities of protons and neutrons to equal values, $n_{n} / n_{p} \simeq\left\langle\sigma_{p \rightarrow n}^{\pi} v\right\rangle /\left\langle\sigma_{n \rightarrow p}^{\pi} v\right\rangle \simeq 1$. $^{1}$ The effect of kaons is qualitatively similar, but leads to a slightly different neutron-to-proton ratio (Appendix D 2).

The impact of this effect on the primordial ${ }^{4} \mathrm{He}$ abundance depends on how long mesons remain present in plasma in significant amounts. Once mesons are created, they can (i) scatter and lose energy, (ii) decay, or (iii) participate in $p \leftrightarrow n$ conversion. The corresponding rates are very different: at $\mathrm{MeV}$ temperatures and below, $\Gamma_{\text {scat }}^{h} \gg$ $\Gamma_{\text {decay }}^{h} \gg \Gamma_{p \leftrightarrow n}^{h}$ (see Ref. [37]). The instantaneous number density of mesons is an interplay between their production (via decays of HNLs) and their decays:

$$
n_{h}^{\text {inst }}=n_{N}(T) \cdot \mathrm{Br}_{N \rightarrow h} \frac{\Gamma_{N, \mathrm{dec}}}{\Gamma_{h, \mathrm{dec}}}=n_{N}(T) \cdot \mathrm{Br}_{N \rightarrow h} \frac{\tau_{h}}{\tau_{N}} .
$$

Here, $\mathrm{Br}_{N \rightarrow h}$ is the branching of HNLs into mesons (Appendix C). $n_{N}(T)$ is the number density of HNLs. We consider here HNLs that were produced thermally and decouple at some temperature $T_{\mathrm{dec}}$ (Appendix B). ${ }^{2}$ Therefore,

\footnotetext{
${ }^{1}$ For each of the processes (1), there are no inverse reactions. Indeed, $\pi^{0}$ decays very fast, whereas $\gamma$ 's quickly lose their energy. Therefore, the conversion (1) is highly nonequilibrium, and the corresponding value of $n_{n} / n_{p}$ is not given by the usual Boltzmann exponent.

${ }^{2}$ Besides thermal production, out-of-equilibrium production mechanisms of HNLs exist (see, e.g., Refs. [41,42]), which we leave for future works (see, however, Ref. [31] where a part of this parameter space was explored).
}

$$
n_{N}(T)=\left(\frac{a_{\mathrm{dec}}}{a(T)}\right)^{3} \cdot n_{N}^{\mathrm{dec}} \cdot e^{-\frac{t(T)}{\tau_{N}}},
$$

where $n_{N}^{\mathrm{dec}}$ is the HNL number density at decoupling, and $a(T)\left(a_{\mathrm{dec}}\right)$ is the scale factor at temperature $T$ (correspondingly, at HNL decoupling).

The number of $p \leftrightarrow n$ reactions per nucleon occurring after time $t \gg \tau_{N}$ [or below some corresponding temperature $T(t)]$ is thus

$$
\begin{aligned}
N_{p \leftrightarrow n}^{h}(T) & =\sum_{h} \int_{t(T)}^{\infty} d t n_{h}^{\mathrm{inst}}(T) \cdot\left\langle\sigma_{p \leftrightarrow n}^{h} v\right\rangle \\
& \approx\left(\frac{a_{\mathrm{dec}}}{a}\right)^{3} \frac{n_{N}^{\mathrm{dec}}}{n_{B}} \cdot e^{-\frac{t(T)}{\tau_{N}}} \cdot \mathrm{Br}_{N \rightarrow h} \cdot P_{\mathrm{conv}},
\end{aligned}
$$

where $n_{B}$ is the baryon number density, the sum goes over meson species, and $P_{\text {conv }}$ is the probability for a single meson to interact with nucleons before decaying:

$$
P_{\text {conv }} \simeq \frac{n_{B} \cdot\left\langle\sigma_{p \leftrightarrow n}^{h} v\right\rangle}{\Gamma_{\text {decay }}^{h}} .
$$

At $\mathcal{O}(1 \mathrm{MeV})$ temperatures, $P_{\text {conv }} \sim 10^{-2}-10^{-1}$; see Appendix D 1.

The meson-driven conversion keeps the value $n_{n} / n_{p} \simeq 1$ roughly until a temperature $T_{0}$ when the number of reactions drops below one,

$$
N_{p \leftrightarrow n}^{h}\left(T_{0}\right) \simeq 1,
$$

and weak SBBN reactions start to relax the $n / p$ ratio down to its SBBN value; see Fig. 1 (left panel). However, if $T_{0}$ is close enough to the freeze-out of weak $p \leftrightarrow n$ processes, occurring at $T_{n} \simeq 0.8 \mathrm{MeV}$, the relaxation is not complete (Fig. 1, right panel). This leads to a positive correction $\Delta\left(n_{n} / n_{p}\right)$ as compared to the SBBN case, which translates to an increase of the ${ }^{4} \mathrm{He}$ abundance $\Delta Y_{p}$.

In this way, the upper bound on the ${ }^{4} \mathrm{He}$ abundance $Y_{p, \max }$ is translated to the lower bound $T_{0} \geq T_{0}^{\min }$. Together with the relations (5)-(7), this allow us to find an upper limit on the HNL lifetime $\tau_{N}$ :

$$
\tau_{N} \lesssim \frac{t\left(T_{0}^{\min }\right)}{\ln \left[\sum_{h}\left(\frac{a_{\mathrm{dec}}}{a_{0}}\right)^{3} \frac{n_{N}^{\mathrm{dec}} P_{\mathrm{con} B} \mathrm{Br}_{N \rightarrow h}}{n_{\gamma}\left(T_{0}^{\mathrm{min}}\right) \eta_{B}}\right]}
$$

Here, $n_{\gamma}$ is the number density of photons, $\eta_{B}$ is the baryonto-photon ratio, and $t(T)$ is the time-temperature relation. $t(T)$ is given by the Standard Model relation $t(T)=\frac{M_{*}}{2 T^{2}}$, where $M_{*}=\frac{M_{\mathrm{Pl}}}{1.66 \sqrt{g_{*}}}$ is the reduced Planck mass, and $g_{*}(T) \simeq 10.6$ for $T \simeq 1-2 \mathrm{MeV}^{3}$

\footnotetext{
${ }^{3}$ This is indeed the case for short-lived HNLs with $\tau_{N} \ll 0.1 \mathrm{~s}$.
} 

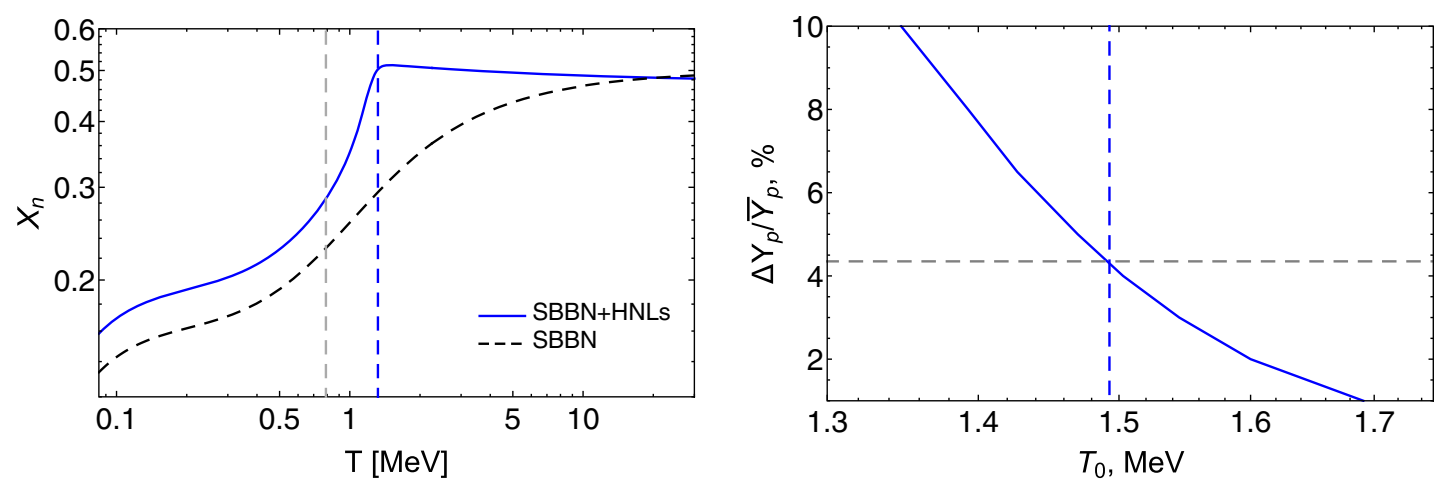

FIG. 1. Left panel: temperature evolution of the neutron abundance $X_{n}=n_{n} /\left(n_{n}+n_{p}\right)$ in the presence of pions from decays of an HNL with mass $m_{N}=400 \mathrm{MeV}$ and lifetime $\tau_{N}=0.03 \mathrm{~s}$. Below $T \simeq 100 \mathrm{MeV}$, pions drive the neutron abundance to $X_{n} \approx 0.5$. At temperatures $T_{0} \simeq 1.3 \mathrm{MeV}$ (the blue vertical dashed line) pions disappear, and $X_{n}$ starts to relax towards its SBBN value but does not reach it. After the neutron decoupling (the gray vertical line) $X_{n}$ evolves mainly due to the neutron decays. Right panel: a relation between the temperature $T_{0}$ [defined by Eq. (7)] and corrections to the ${ }^{4} \mathrm{He}$ abundance, as compared to the SBBN central value $\bar{Y}_{p} \approx 0.247$. It corresponds to the case of when only charged pions are present in plasma. The gray horizontal line corresponds to the maximally allowed correction $\Delta Y_{p} / \bar{Y}_{p}=4.35 \%$ that we adopt in this work (see Appendix A). The intersection of the gray and colored lines defines the temperature $T_{0}^{\mathrm{min}}$. Its actual value in the presence of all mesons always lies close to $T_{0}^{\mathrm{min}} \approx 1.5 \mathrm{MeV}$ independently of the HNL mass (see Appendix D 2).

Let us rewrite the logarithmic factor in Eq. (8) as

$$
\left(\frac{a_{\mathrm{dec}}}{a_{0}}\right)^{3} \frac{n_{N, \mathrm{dec}}}{n_{\gamma}\left(T_{0}^{\min }\right)}=\frac{n_{N, \mathrm{dec}}}{n_{\gamma}\left(T_{\mathrm{dec}}\right)} \cdot\left(\frac{a_{\mathrm{dec}} T_{\mathrm{dec}}}{a_{0} T_{0}^{\min }}\right)^{3} .
$$

HNLs with $m_{N} \gtrsim m_{\pi}$ and lifetimes $\tau_{N} \ll 0.1 \mathrm{~s}$ decouple while being ultrarelativistic, $T_{\mathrm{dec}} \gg m_{N}$ (see Appendix B), implying $n_{N, \mathrm{dec}} / n_{\gamma}\left(T_{\mathrm{dec}}\right) \approx 3 / 2$. In SBBN at temperatures $T \gtrsim 1 \mathrm{MeV}$, all particles are at local equilibrium, which define the dynamics of the scale factor:

$$
\left(\frac{a_{\mathrm{dec}} T_{\mathrm{dec}}}{a_{0} T_{0}^{\min }}\right)^{3} \approx \frac{g_{*}\left(T_{0}^{\mathrm{min}}\right)}{g_{*}\left(T_{\mathrm{dec}}\right)} \simeq \frac{1}{8} .
$$

Decays of heavy HNLs violate thermal equilibrium at $\mathcal{O}(1 \mathrm{MeV})$ and the scaling (10) is not valid. This leads to an additional decrease of this ratio by a factor of $0.1-0.6$ for HNL masses $m_{\pi} \lesssim m_{N} \lesssim 3 \mathrm{GeV}$ (we will use $\frac{1}{3}$ for normalization below); see Appendix B.

This results in

$$
\tau_{N} \lesssim \frac{0.023\left(\frac{1.5 \mathrm{MeV}}{T_{0}^{\min }}\right)^{2} \mathrm{~s}}{1+0.07 \ln \left[\frac{P_{\text {conv }}}{0.1} \frac{\mathrm{Br}_{N \rightarrow h}}{0.4} \frac{2 n_{N, \mathrm{dec}}}{3 n_{\gamma}\left(T_{\mathrm{dec}}\right)} \cdot 24\left(\frac{a_{\mathrm{dec}} T_{\mathrm{dec}}}{a_{0} T_{0}^{\min }}\right)^{3}\right]}
$$

Using values of $\mathrm{Br}_{N \rightarrow h}, P_{\text {conv }}$, and the scale factors ratio (Appendixes C, D 1, and B, respectively), we conclude that the logarithm term in Eq. (11) is $\mathcal{O}(1)$ for HNLs in the mass range $m_{N}=\mathcal{O}(1 \mathrm{GeV})$ and affects the overall bound very weakly. Therefore, the bound depends only on $T_{0}^{\min }$.

The presence of mesons increases the ${ }^{4} \mathrm{He}$ abundance. Therefore, to fix $T_{0}^{\min }\left(m_{N}\right)$ we need to adopt an upper bound on the primordial ${ }^{4} \mathrm{He}$ abundance, $Y_{p \text {, max }}$, that is consistent with measurements [43]. The smallest error bars come from measuring $Y_{p}$ in low-metallicity interstellar regions and extrapolating its value to zero metallicity (pioneered in Ref. [44]). Several groups [10-14] have determined $Y_{p}$ using this method, albeit with different data and assumptions. The resulting scatter between results is larger than the reported error bars. We treat this difference as an additional systematic uncertainty and adopt the maximal value $Y_{p, \max }=0.2573$ (see Appendix A). The maximally allowed relative deviation is therefore

$$
\Delta Y_{p} / Y_{p, \mathrm{SBBN}} \approx 4.35 \% \text {. }
$$

To relate $\Delta Y_{p}$ and $T_{0}^{\mathrm{min}}$, we study how the $n_{n} / n_{p}$ ratio is relaxed below $T_{0}$. The relaxation occurs solely via the SBBN reaction,

$\frac{d X_{n}}{d t}=\Gamma_{p \rightarrow n}^{\mathrm{SBBN}}\left(1-X_{n}\right)-\Gamma_{n \rightarrow p}^{\mathrm{SBBN}} X_{n}, \quad X_{n}=\frac{n_{n}}{n_{n}+n_{p}}$,

albeit with the altered initial condition $X_{n}\left(T_{0}\right)=X_{n}^{h} \simeq 1 / 2$. $\left[\Gamma_{p \leftrightarrow n}^{\mathrm{SBBN}}(t)\right.$ are SBBN rates; see Ref. [15].] A non-SBBN value of $X_{n}\left(T_{0}\right)$ is the dominant effect of short-lived HNLs on $Y_{p}$. At temperatures $T \lesssim T_{0}$, for HNLs with lifetimes $\tau_{N} \lesssim 0.02 \mathrm{~s}$, all other quantities that are relevant for BBN dynamics $-\eta_{B}$, time-temperature relation, the nuclear reactions chain-remain the same as in SBBN, which is because most of HNLs are no longer left in the plasma at these temperatures (see also Appendix D 2). As a result, a value of $X_{n}\left(T_{0}\right)$ is translated into $\Delta Y_{p}$ via 

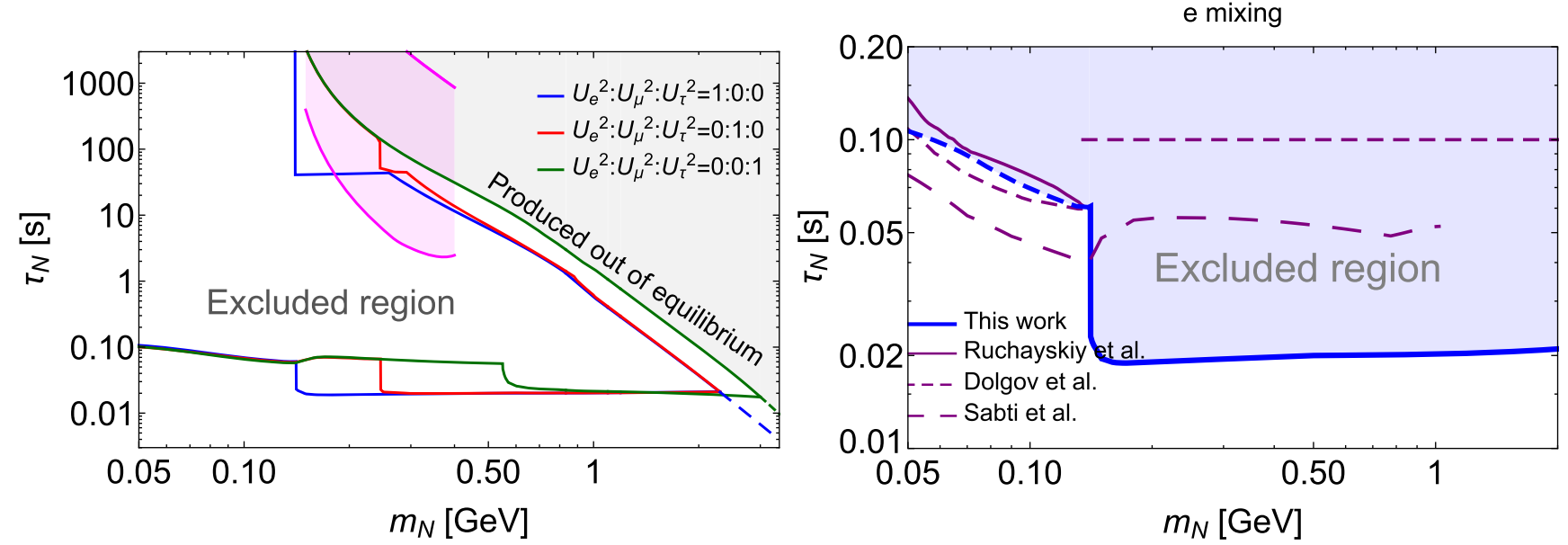

FIG. 2. Left panel: BBN bounds on the HNL lifetime for different mixing patterns. The gray region is excluded as a result of this work (for masses below the pion threshold we use the results of Ref. [49]). The magenta shaded region corresponds to the domain excluded in Ref. [31]. We consider HNLs that are produced thermally and sufficiently short lived, such that they do not survive until the onset of nuclear reactions (Appendix E). Right panel: comparison of the results of this work (thick blue line) with the results of the previous works [22,25,32] (purple lines) assuming mixing with electron flavor only. Notice that other works adopted different values for the maximally admissible ${ }^{4} \mathrm{He}$ abundance when deriving their bounds: $Y_{p, \max }=0.2696$ in Refs. [22,25] and $Y_{p, \max }=0.253$ in Ref. [32], as compared to $Y_{p, \max }=0.2573$ in this work (see text for details).

$$
\frac{\Delta Y_{p}}{Y_{p, \mathrm{SBBN}}}=\frac{\Delta X_{n}\left(T_{\mathrm{BBN}}\right)}{X_{n, \mathrm{SBBN}}\left(T_{\mathrm{BBN}}\right)}
$$

where $T_{\mathrm{BBN}} \approx 84 \mathrm{keV}$ is the temperature of the onset of nuclear reactions in SBBN [15]. The maximal admissible correction (12) is reached for $T_{0}^{\min }=1.50 \mathrm{MeV}$, almost independently of HNL mass (see Fig. 1 and Appendix D 2). Plugging $T_{0}^{\min }=1.50 \mathrm{MeV}$ into Eq. (11), we get our final limit

$$
\tau_{N} \lesssim 0.023 \mathrm{~s}
$$

To obtain the bound (11), we consider exclusively mesondriven $p \leftrightarrow n$ processes for $T>T_{0}^{\min }$ and only weak SBBN processes for $T<T_{0}^{\min }$. We also numerically solve Eq. (13) for the neutron abundance in the presence of both mesondriven and SBBN $p \leftrightarrow n$ conversion rates in Appendix D 2, and obtain constraints at the level of 0.019-0.021 s, in perfect agreement with the bound (15). We also repeat our analysis for the case of the GeV-mass scalar that mixes with the Higgs and find an excellent agreement with Refs. [34,35].

Our analysis remains valid until $\tau_{N}$ reaches $\mathcal{O}(40 \mathrm{~s})$. HNLs with longer lifetimes survive until the onset of the nuclear reactions. Mesons from their decays may then dissociate already formed nuclei, driving $Y_{p}$ down again; see Appendix E. This effect has been analyzed in Ref. [45], demonstrating that such long-lived HNLs are also excluded.

\section{CONCLUSION}

We demonstrated that HNLs with semileptonic decay channels significantly affect the primordial ${ }^{4} \mathrm{He}$ abundance, as mesons from their decays drive the $p \leftrightarrow n$ conversion rates away from their SBBN values (cf. Refs. [33-35]). In order to avoid ${ }^{4} \mathrm{He}$ overproduction, mesons should disappear from the primordial plasma by $T=T_{0}^{\min } \simeq 1.50 \mathrm{MeV}$. The neutron abundance will then have enough time to relax down to its SBBN value before the onset of deuteron formation. These requirements severely constrain the parameter space of the HNLs with $0.023 \mathrm{~s} \leq \tau_{N} \leq 40 \mathrm{~s}$ for masses $m_{N}>140 \mathrm{MeV}$. Accidentally, HNLs with $\tau_{N} \gtrsim 40 \mathrm{~s}$ are also excluded [45,46], but we did not consider this case here.

We show our results for the case of two nearly-degenerate in mass HNLs that entered in thermal equilibrium and then froze out (which is reflected in both abundance calculation and decay width/patterns), as motivated by the Neutrino Minimal Standard Model (or $\nu$ MSM) (see, e.g., Refs. $[6,47,48]$ ). The final bounds for different mixing patterns are shown in Figs. 2 and 3. Our constraints can be generalized to other HNL models; see, e.g., Ref. [45].

Confronted with the bounds from accelerator searches, we ruled out HNLs with mass below $500 \mathrm{MeV}$ (for electron mixing) and $350 \mathrm{MeV}$ (for muon mixing). Moreover, a tighter bound means that future searches at Intensity Frontier (specifically, the SHiP experiment [51]) can reach the BBN bottom line and completely rule out HNLs with masses up to $750 \mathrm{MeV}$, which was not the case before (see, e.g., Refs. $[8,57])$.

The comparison with the previous results $[22,25,32]$ is shown in Fig. 2 (right panel). Our bound (15) is a factor of $\sim 5$ stronger than the previous result [22]. The recent reanalysis [32] did not take into account the effects of mesons, and therefore their results are a factor of 2-3 less conservative. 

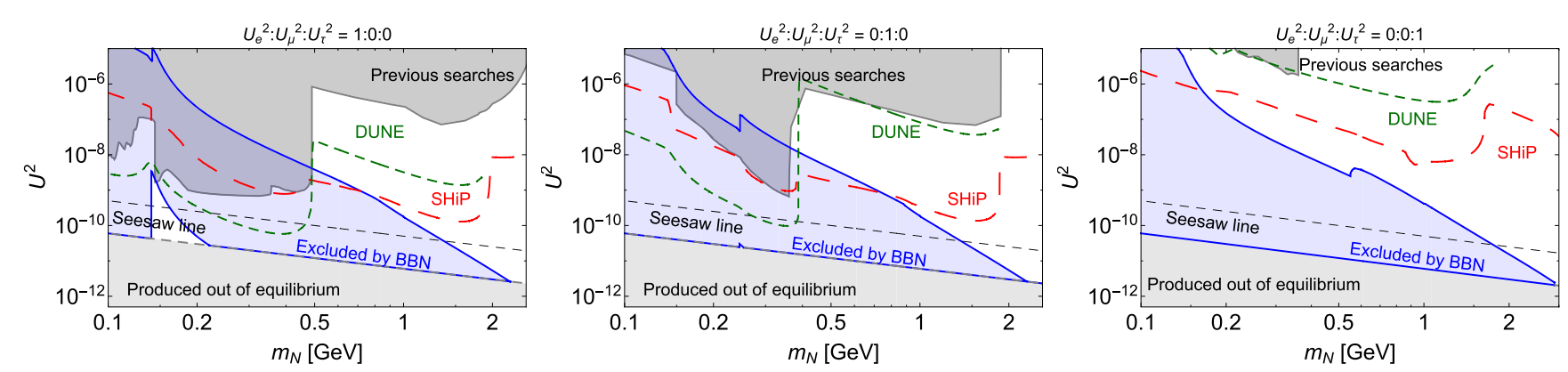

FIG. 3. Bounds for HNLs mixed with a particular flavor. The blue area is excluded by our present analysis combined with Ref. [49] (for HNL masses below the charged pion production threshold). The dark gray area denotes the excluded HNL parameter space from previous searches [1], including the latest NA62 search [50]. The red and greed dashed lines show the sensitivity of several future Intensity Frontier experiments with the highest sensitivity in the regions of interest: SHiP [51,52] and DUNE [53-55] (see Ref. [8]). Finally, the black dashed line denotes the seesaw bound applicable if two degenerate-in-mass HNLs are responsible for neutrino oscillations (as in the $\iota \mathrm{MSM}$ ) [1,56]. The lower bound of the blue region shows the limit of the applicability of our approach: we only consider HNLs that are produced thermally and are sufficiently short lived such that they do not change the nuclear reaction framework by their meson decay products.

The clear qualitative effect discussed in this paper not only leads to a tighter bound on the HNL lifetime and provides a reachable goal for experimental searches, but also allows for an analytic description, unusual in the realm of BBN predictions driven by sophisticated numerical codes.

\section{ACKNOWLEDGMENTS}

We thank K. Bondarenko, N. Sabti for useful discussions, and Yu. Izotov for discussions related to the uncertainties of primordial helium determination. This project has received funding from the European Research Council (ERC) under the European Union's Horizon 2020 research and innovation programme (GA 694896) and from the Carlsberg Foundation.

\section{APPENDIX A: PRIMORDIAL HELIUM ABUNDANCE}

Over the last 6 years five works determined the primordial ${ }^{4} \mathrm{He}$ abundance from stellar measurements [10-14].

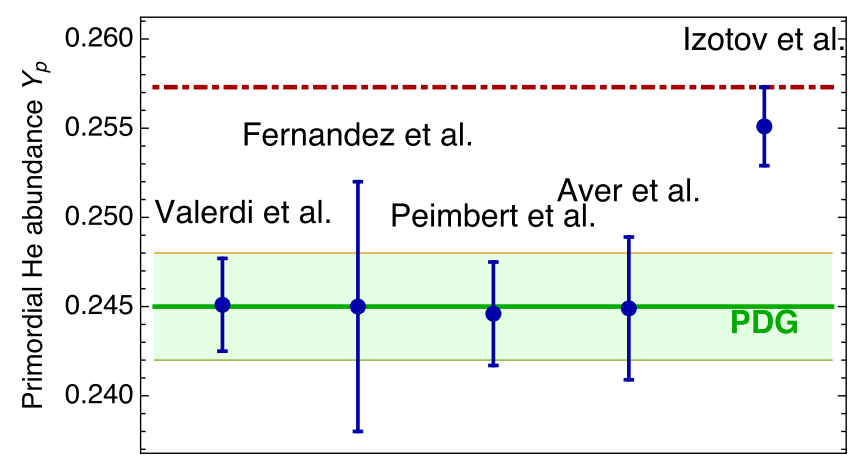

FIG. 4. Measurements of $Y_{p}$ from recent works [10-14]. The green shaded region is the PDG recommended value [43] (with $\pm 1 \sigma$ ). The gray dashed line denotes the $\mathrm{SBBN}$ prediction $\bar{Y}_{p}=$ 0.247 from Ref. [15]. The red dash-dotted line is the maximal admissible value $Y_{p \text {,max }}$ on which we base our analysis.
The formal statistical errors of $Y_{p}$ are at the level of 1-3\%; however, the scatter between different groups is larger (see Fig. 4).

All of these works determined the astrophysical helium abundance through measurements of recombination emission lines of ${ }^{4} \mathrm{He}$ and $\mathrm{H}$ in the metal-poor extragalactic ionized regions, then linearly extrapolating the measurements to zero metallicity. Given the high precision of the results, it is important to take into account various smaller effects: including ${ }^{4} \mathrm{He}$ fluorescent emission, different ion temperatures, spatial temperature fluctuations, and others [58,59]. Additionally, while it is true that the metallicity and helium abundance are positively correlated, the linear extrapolation to zero metallicity may be prone to systematic uncertainties.

The value of $Y_{p}$ predicted within the framework of SBBN is $\bar{Y}_{p}=0.24709 \pm 0.00019$ (see, e.g., Ref. [15]). The effect of mesons leads to an increase of $Y_{p}$ as compared to the SBBN value. Therefore, in order to get a conservative upper bound we assume that the maximally allowed $Y_{p}$ is given by the $1 \sigma$ deviation from the maximal value predicted in Refs. [10-14], which is $Y_{p \text {, } \max }=0.2573$. Note that this upper value significantly deviates from the PDG recommended value [43] $Y_{p, \max }=0.248$ at $1 \sigma$. This translates to the bound

$$
\frac{\Delta Y_{p}}{\bar{Y}_{p}}<4.35 \%
$$

\section{APPENDIX B: EVOLUTION OF HNLS IN THE EARLY UNIVERSE}

In this work we consider HNLs that have entered thermal equilibrium in the early Universe and then froze out. Below we provide necessary details of their evolution, to make our 
presentation more self-contained [49]. The evolution of HNLs with masses above the pion production threshold proceeds in three stages:

(1) The freeze-out of HNLs occurs at some temperature $T_{\text {dec }}$ defined via

$$
\Gamma_{N}^{\mathrm{int}}\left(T_{\mathrm{dec}}\right) \simeq H\left(T_{\mathrm{dec}}\right)
$$

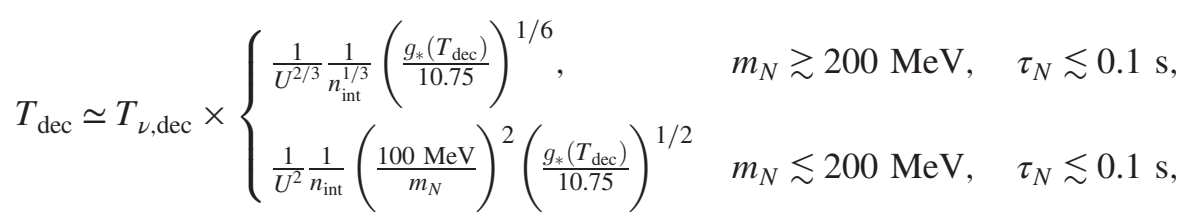

where $T_{\nu, \mathrm{dec}} \approx 1.4 \mathrm{MeV}$ is the decoupling temperature of active neutrinos, and the parameter $n_{\text {int }}=$ $\frac{\Gamma_{N}^{\text {int }}}{U^{2} G_{F}^{2} T^{5}}$ varies from $\simeq 1.5$ at $T \simeq \mathcal{O}(1 \mathrm{MeV})$ to $\simeq 10$ at $\mathrm{GeV}$ temperatures (for Dirac HNLs). According to this estimate, for lifetimes $\tau_{N} \sim 0.01-0.1 \mathrm{~s}$, HNLs decouple while being nonrelativistic for $m_{N} \lesssim$ $200 \mathrm{MeV}$ and relativistic otherwise. To improve this estimate, we solve the following equation:

$\frac{d n_{N}}{d t}+3 H_{\mathrm{SM}}(t) \cdot n_{N}=-\Gamma_{N}^{\mathrm{int}}(T)\left(n_{N}-n_{N, \mathrm{eq}}\right)$,

where $n_{N, \text { eq }}$ is the number density of HNLs at equilibrium (i.e., calculated using the Fermi-Dirac distribution). Expressing the total HNL number density in terms of the abundance $Y_{N}$,

$$
Y_{N}=\left(\frac{n_{N}}{s}\right)_{T=T_{\mathrm{dec}}}
$$

where $s=g_{*} \frac{2 \pi^{2}}{45} T^{3}$ is the entropy density, we find $Y_{N} \simeq \frac{0.6}{g_{*}\left(T_{-}\right)}$in the ultrarelativistic regime and a factor of $\mathcal{O}(2)$ larger otherwise; see Fig. 5.

After the freeze-out, the comoving number density of HNLs changes only due to HNL decays. The physical number density thus evolves as

$$
n_{N}(T)=n_{N}\left(T_{\mathrm{dec}}\right) \cdot\left(\frac{a\left(T_{\mathrm{dec}}\right)}{a(T)}\right)^{3} \cdot e^{-t / \tau_{N}} .
$$

(2) Decays of HNLs inject energy into the primordial plasma. This changes the time-temperature relation and the scale factor evolution as compared to SBBN. The HNL decays provide additional dilution of any

\footnotetext{
${ }^{4}$ We use the matrix elements of all relevant processes with HNLs from Ref. [32].
}

where $\Gamma_{N}^{\text {int }}$ is a rate of processes that keep HNLs in thermal equilibrium with the primordial plasma, ${ }^{4}$ and the Hubble rate $H$ corresponds to its Standard Model value (at the decoupling, HNLs with masses $m_{N} \gtrsim m_{\pi}$ and lifetimes in the range of interest contribute just a small fraction of the energy density of the Universe). Within $\mathcal{O}(1)$ accuracy, the decoupling temperature $T_{\text {dec }}$ may be estimated as

decoupled relics (including themselves) in comparison to the SBBN case:

$$
\zeta=\left(\frac{a_{\mathrm{SBBN}}}{a_{\mathrm{SBBN}+\mathrm{N}}}\right)^{3}<1,
$$

where $a_{\mathrm{SBBN}}^{-1}(T) \propto g_{*}^{1 / 3} T$ is the scale factor in SBBN, and the scale factors are evaluated at times $t \gg \tau_{N}$. To calculate $\zeta$, we solve the Friedmann equation under the assumption that neutrinos are in perfect equilibrium and we neglect the mass of electrons:

$$
\begin{aligned}
H^{2}(t) & =\frac{1}{M_{\mathrm{Pl}}^{2}} \frac{8 \pi}{3}\left[\rho_{\mathrm{rad}}+m_{N} \cdot n_{N}(T)\right], \\
4 \frac{\rho_{\mathrm{rad}}}{T} \frac{d T}{d t} & =\frac{m_{N} n_{N}(t)}{\tau_{N}}-4 H(t) \cdot \rho_{\mathrm{rad}},
\end{aligned}
$$

where the number density of HNLs is given by Eq. (B5). This is a reasonable assumption, since most of the HNLs with lifetimes $\tau_{N} \ll 0.1 \mathrm{~s}$ decay much earlier than neutrinos decouple.

(3) Strong meson effects mean that we need to trace the number of HNLs even at times $t \gg \tau_{N}$ :

$$
\begin{aligned}
n_{N}\left(t \gg \tau_{N}\right)= & n_{N}\left(T_{-}\right) \cdot\left(\frac{a_{\mathrm{SBBN}}\left(T_{-}\right)}{a_{\mathrm{SBBN}}(T)}\right)^{3} \\
& \cdot \zeta \cdot e^{-t(T) / \tau_{N} \approx 0.4 Y_{N} \cdot g_{*, \mathrm{SBBN}} T^{3}} \\
& \cdot \zeta \cdot e^{-t(T) / \tau_{N}},
\end{aligned}
$$

where $t(T)$ is the same as in SBBN. ${ }^{5}$ Because of the suppression, the effect of this population on the expansion of the Universe may be neglected. However, this exponential tail may still produce mesons

\footnotetext{
${ }^{5}$ At times $t \gg \tau_{N}$, the time-temperature relation differs from SBBN only by the value of $N_{\text {eff }}$. However, the latter may change only if neutrinos are not in perfect equilibrium, and hence $t(T)$ is the same as in SBBN for lifetimes $\tau_{N} \ll 0.1 \mathrm{~s}$.
} 

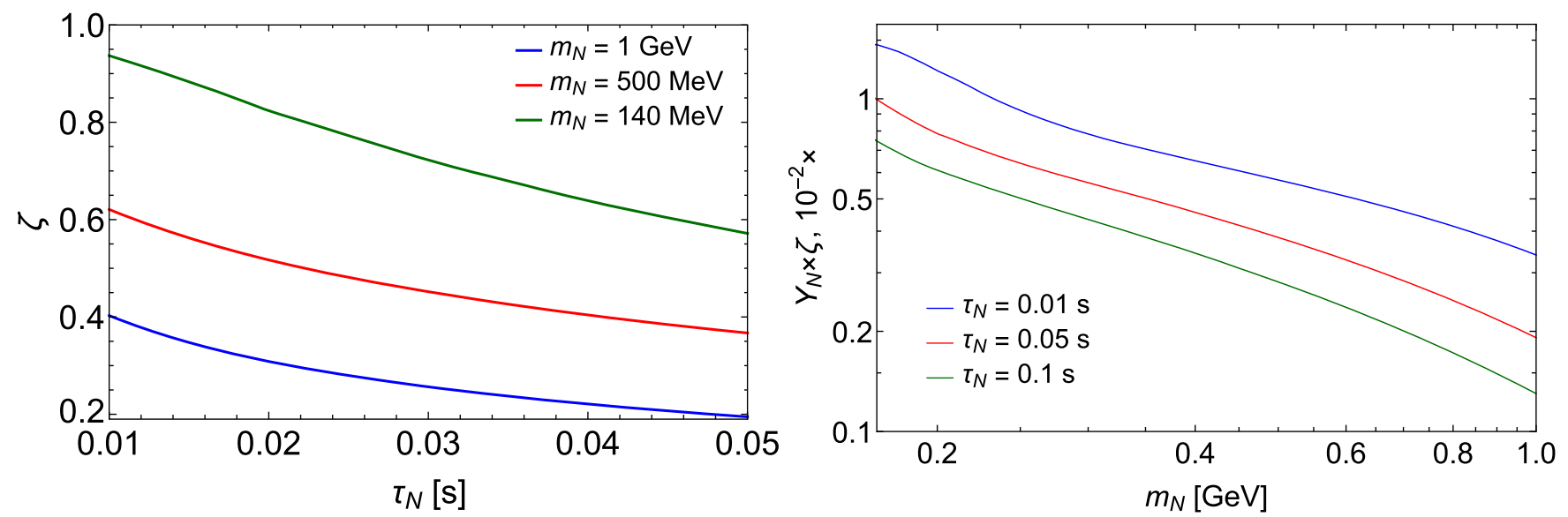

FIG. 5. Left panel: dilution factor (B6) for short-lived HNLs mixing with $\nu_{e}$. Right panel: HNL abundance times dilution factor as a function of mass for particular values of the lifetime. Details of the calculation of the abundances and $\zeta$ are given in Ref. [49]. The dilution factor is calculated when most of the HNLs have decayed and do not contribute to the entropy density. Note that we define the abundance at the moment of decoupling, and hence it does not change with decays.

in amounts sufficient to change the dynamics of the $n / p$ ratio.

The values of the HNL abundance and the dilution factor versus its mass and lifetime are given in Fig. 5.

\section{APPENDIX C: HADRONIC DECAYS OF HNLS}

In this work we consider a pair of HNLs, degenerate in mass and having similar mixing angles. Two such HNLs form a single quasi-Dirac fermion $[60,61]$. The abundance of a meson $h$ produced from such HNLs is proportional to the quantity $Y_{N} \cdot \mathrm{Br}_{N \rightarrow h}$. The mass dependence of $\mathrm{Br}_{N \rightarrow h}$ for different mesons $h$ and mixing patterns is shown in Fig. 6. We are interested only in the abundances of light mesons (pions and kaons) and for HNL masses well above pion/kaon thresholds we should account for "secondary mesons." This is discussed below, mainly following Ref. [40].

\section{Decays into pions}

In the case of the pure $e / \mu$ mixings, the charged pion production threshold corresponds to $m_{N}=m_{\pi}+m_{l}$, where $l=e / \mu$. For $\tau$ mixing, the similar charged-currentmediated channel opens up only at $m_{N}=m_{\tau}+m_{\pi} \simeq$ $1.9 \mathrm{GeV}$. However, for all types of mixings charged pions may appear as secondary particles in decays of neutral mesons,

$N \rightarrow h^{0}+\nu_{\alpha}, \quad h^{0} \rightarrow \pi^{ \pm}+X, \quad$ where $h^{0}=\rho^{0}, \eta^{0}, \eta^{\prime}, \omega^{0}, \phi$.

Therefore, for $\tau$ mixing charged pions may appear at masses $m_{N} \geq m_{\eta^{0}}$. We use the branching ratios $\mathrm{Br}_{\eta^{0} \rightarrow \pi^{ \pm} X} \approx$ $0.27, \mathrm{Br}_{\rho^{0, \pm} \rightarrow \pi^{ \pm} X} \approx 1$ [43].

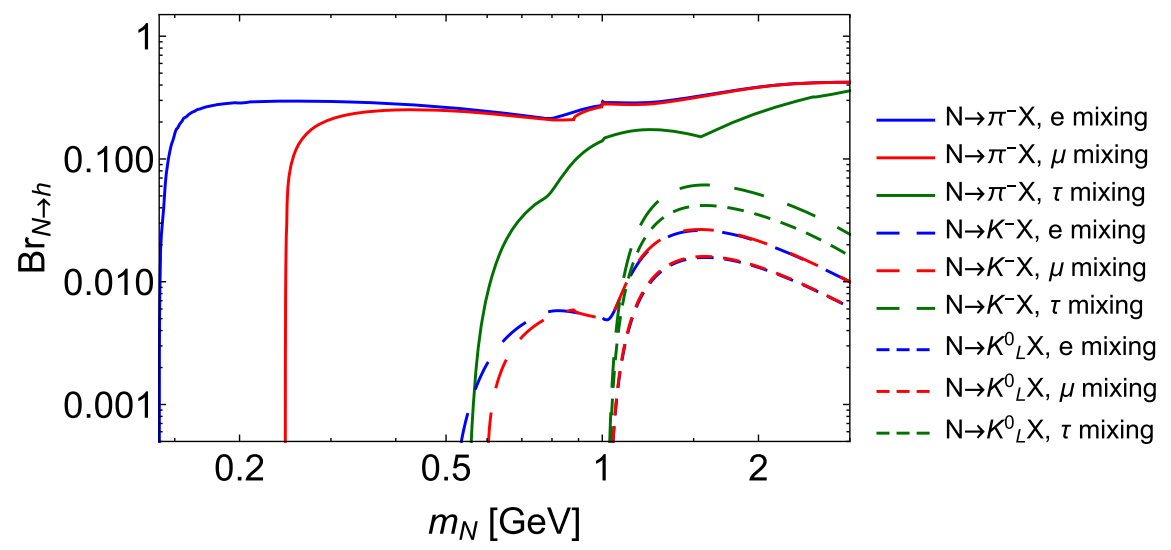

FIG. 6. Branching ratios of HNL decays into mesons $h=\pi^{-}, K^{-}, K_{L}^{0}$. Secondary decays are also included (see text for details). 
Above $m_{N} \simeq 1 \mathrm{GeV}$, decays of HNLs into pions cannot be approximated by single meson decays. Indeed, decays of $\mathrm{GeV}$ mass range HNLs are similar to decays of $\tau$ lepton [40], whereas for the latter hadronic decays are dominated by multi-pion channels [43]. We estimate the width of multi-pion decays as the difference between the total width into quarks and the width into single mesons:

$$
\Gamma_{N \rightarrow n \pi}=\Gamma_{N \rightarrow \text { quarks }}-\sum_{h=\pi, K, \rho, \ldots} \Gamma_{N \rightarrow h X} .
$$

For multiplicities $\mathcal{N}$ of decays of HNLs into charged pions $N \rightarrow \pi^{ \pm}$(i.e., the amount of $\pi^{ \pm}$per multi-hadronic decay of HNLs), we will use multiplicities for multihadronic decays of $\tau$ leptons. Namely, $\mathcal{N}_{N \rightarrow \pi^{+}}=\mathcal{N}_{\tau^{+} \rightarrow \pi^{+}} \approx 1.35$, $\mathcal{N}_{N \rightarrow \pi^{-}}=\mathcal{N}_{\tau^{+} \rightarrow \pi^{-}} \approx 0.34$. The effective branching into $\pi^{-}$ from multi-pion decays is

$$
\begin{aligned}
\operatorname{Br}_{N \rightarrow \pi^{-}}^{\text {multion }} & =\mathcal{N}_{N \rightarrow \pi^{-}} \cdot \frac{\Gamma_{N \rightarrow n \pi}}{\Gamma_{N}}, \\
\operatorname{Br}_{\bar{N} \rightarrow \pi^{-}}^{\text {multion }} & =\mathcal{N}_{\bar{N} \rightarrow \pi^{-}} \cdot \frac{\Gamma_{N \rightarrow n \pi}}{\Gamma_{N}} .
\end{aligned}
$$

Since the bound on the meson driven $p \leftrightarrow n$ conversion is only logarithmically sensitive to the value of $\mathrm{Br}_{N \rightarrow \pi^{ \pm}}$, our results depend on these assumptions weakly.

\section{Decays into kaons}

Below $m_{N}=m_{\phi}$, charged kaons may appear only through the mixing with $e / \mu$ in the process $N \rightarrow K^{-} l$. This decay is Cabibbo suppressed [40] and almost 2 orders of magnitude smaller than into pions. Neutral kaons appear only in the final states with three or more particles (such as $N \rightarrow K^{0}+\bar{K}^{0}+\nu_{\alpha}$ and $N \rightarrow K^{+}+\bar{K}^{0}+\ell^{-}$, etc.).

HNLs heavier than the $\phi$ meson may produce both charged and neutral kaons via decays $N \rightarrow \phi \nu, \phi \rightarrow K K$. We assume that $K^{0}$ contains equal admixtures of $K_{L}^{0}$ and $K_{S}^{0}$, i.e., $\mathrm{Br}_{N \rightarrow K_{L}^{0}}=\mathrm{Br}_{N \rightarrow K^{0}} / 2$. We use the branching ratios $\mathrm{Br}_{\phi \rightarrow K^{-}} \approx 0.5, \mathrm{Br}_{\phi \rightarrow K_{L}^{0}} \approx 0.34$ [43].

\section{APPENDIX D: CHANGES IN $p \leftrightarrow n$ RATES DUE TO THE PRESENCE OF MESONS}

In this Appendix, we provide details on our estimate of the effect of mesons on BBN.

\section{Processes and cross sections}

\section{a. Pions}

The thresholdless processes with charged pions are

$$
\pi^{-}+p \rightarrow n+\pi^{0} / \gamma, \quad \pi^{+}+n \rightarrow p+\pi^{0} .
$$

The cross sections at threshold are [37]

$$
\begin{aligned}
& \left\langle\sigma_{p \rightarrow n}^{\pi^{-}} v\right\rangle \approx 4.3 \times 10^{-23} F_{c}^{\pi}(T) \mathrm{m}^{3} / \mathrm{s}, \\
& \frac{\left\langle\sigma_{p \rightarrow n}^{\pi^{-}} v\right\rangle}{\left\langle\sigma_{n \rightarrow p}^{\pi^{+}} v\right\rangle} \approx 0.9 F_{c}^{\pi}(T)
\end{aligned}
$$

where $F_{c}^{h}$ is the Sommerfeld enhancement of the cross section due to the presence of two oppositely charged particles in the in-state:

$$
F_{c}^{h}=\frac{x}{1+e^{-x}}, \quad \text { where } x=\frac{2 \pi \alpha_{\mathrm{EM}}}{v_{e}}
$$

where $v_{e} \approx \sqrt{\frac{T}{m_{h}}}+\sqrt{\frac{T}{m_{p}}}$ is the relative velocity between a nucleon and a meson. $F_{c}$ is of order of one at $T \simeq 1 \mathrm{MeV}$.

\section{b. Kaons}

The thresholdless $n \leftrightarrow p$ conversions driven by kaons are

$$
\begin{aligned}
K^{-}+p & \rightarrow \Sigma^{ \pm / 0} / \Lambda+\pi^{\mp / 0} / \pi^{0} \rightarrow n+2 \pi, \\
K^{-}+n & \rightarrow \Sigma^{-/ 0} / \Lambda+\pi^{0 /-} / \pi^{-} \rightarrow n+2 \pi, \\
\bar{K}_{L}^{0}+p & \rightarrow \Sigma^{0 /+} / \Lambda+\pi^{+/ 0} / \pi^{+} \rightarrow n+2 \pi, \\
\bar{K}_{L}^{0}+n & \rightarrow \Sigma^{ \pm / 0} / \Lambda+\pi^{\mp / 0} / \pi^{0} \rightarrow p+2 \pi,
\end{aligned}
$$

where $\Lambda, \Sigma$ are the lightest strange hadronic resonances [33].

Their effect is similar to that of pions, but with small differences. (i) The cross sections of the above reactions are higher than the cross sections of (1). ${ }^{6}$ (ii) There is no isotopic symmetry: $K^{+}$mesons do not contribute to $p \leftrightarrow n$ conversion, since there are no thresholdless processes $n+K^{+} \rightarrow p+X$. Indeed, the process $n+K^{+} \rightarrow$ $p+K^{0}$ has the threshold $Q \approx 2.8 \mathrm{MeV}$, while the thresholdless processes going through $s$-quark resonances, similar to Eq. (D4), would require resonances with negative strangeness and positive baryon number, which do not exist. (iii) Neutral kaons do not lose energy before decaying (however, we follow Ref. [33] and approximate the cross sections by threshold values).

The threshold cross sections are

$$
\begin{aligned}
& \left\langle\sigma_{p \rightarrow n}^{K^{-}} v\right\rangle \approx 9.6 \times 10^{-22} F_{c}^{K}(T) \mathrm{m}^{3} / \mathrm{s}, \\
& \frac{\left\langle\sigma_{p \rightarrow n}^{K^{-}} v\right\rangle}{\left\langle\sigma_{n \rightarrow p}^{K^{-}} v\right\rangle} \approx 2.46 F_{c}^{K}(T),
\end{aligned}
$$

\footnotetext{
${ }^{6}$ The reason is that these reactions have higher available phase space and go through hadronic resonances.
} 


$$
\left\langle\sigma_{p \rightarrow n}^{K^{0}} v\right\rangle \approx 1.95 \times 10^{-22} \mathrm{~m}^{3} / \mathrm{s}, \quad \frac{\left\langle\sigma_{p \rightarrow n}^{K_{L}^{0}} v\right\rangle}{\left\langle\sigma_{n \rightarrow p}^{K_{L}^{0}} v\right\rangle} \approx 0.41
$$

\section{c. Conversion probabilities}

A probability for a meson $h$ to convert $p \leftrightarrow n$ before decaying is given by

$$
P_{\text {conv }}^{h} \approx \frac{\left\langle\sigma_{p \leftrightarrow n}^{h} v\right\rangle n_{B}}{\Gamma_{\text {decay }}^{h}},
$$

where $\Gamma_{\text {decay }}^{h}$ is the decay width and $n_{B}$ is the baryon number density. The decay widths of mesons are [43]

$$
\Gamma_{\text {decay }}^{\pi^{ \pm}} \approx 3.8 \times 10^{7} \mathrm{~s}^{-1}, \quad \Gamma_{\text {decay }}^{K^{-}} \approx 8.3 \times 10^{7} \mathrm{~s}^{-1}, \quad \Gamma_{\text {decay }}^{K_{L}^{0}} \approx 2 \times 10^{7} \mathrm{~s}^{-1} .
$$

Using Eqs. (D2), (D5), and (D8), for the $p \rightarrow n$ conversion probabilities we obtain

$$
P_{\text {conv }}^{\pi^{-}}(T) \approx 2.5 \times 10^{-2}\left(\frac{T}{1 \mathrm{MeV}}\right)^{3}, \quad P_{\text {conv }}^{K^{-}}(T) \approx 2.8 \times 10^{-1}\left(\frac{T}{1 \mathrm{MeV}}\right)^{3}, \quad P_{\mathrm{conv}}^{K_{L}^{0}}(T) \approx 1.6 \times 10^{-1}\left(\frac{T}{1 \mathrm{MeV}}\right)^{3} .
$$

The largeness of the probabilities is caused by the fact that the decay of mesons proceeds through weak interactions, while the $p \leftrightarrow n$ conversion is mediated by strong interactions. In particular, at $T \gtrsim 2 \mathrm{MeV}$ kaons participate in the conversion faster than they decay.

\section{Numeric study}

To verify the analytic estimate (15), we numerically solve the equation for the neutron abundance $X_{n}$, where we include both weak conversion $p \leftrightarrow n$ processes and the meson-driven processes (D1)-(D4). The system of equations has the form

$$
\left\{\begin{array}{l}
\frac{X_{n}}{d t}=\left(\frac{d X_{n}}{d t}\right)_{\mathrm{SM}}+\left(\frac{d X_{n}}{d t}\right)_{\pi}+\left(\frac{d X_{n}}{d t}\right)_{K^{-}}+\left(\frac{d X_{n}}{d t}\right)_{K_{L}^{0}}, \\
\frac{d n_{\pi^{-}}}{d t}=n_{N} \frac{\mathrm{Br}_{N_{\pi^{-}}}}{\tau_{N}}-\Gamma_{\text {decay }}^{\pi^{-}} n_{\pi^{-}}-\left\langle\sigma_{p \rightarrow n}^{\pi^{-}} v\right\rangle\left(1-X_{n}\right) n_{B} n_{\pi^{-}}, \\
\frac{d n_{\pi^{+}}}{d t}=n_{N} \frac{\mathrm{Br}_{N \rightarrow \pi^{+}}}{\tau_{N}}-\Gamma_{\text {decay }}^{\pi^{+}} n_{\pi^{+}}-\left\langle\sigma_{n \rightarrow p}^{\pi^{+}} v\right\rangle X_{n} n_{B} n_{\pi^{+}}, \\
\frac{d n_{K^{-}}}{d t}=n_{N} \frac{\operatorname{Br}_{N \rightarrow K^{-}}}{\tau_{N}}-\Gamma_{\text {decay }}^{K^{-}} n_{K^{-}}-\left\langle\sigma_{p \rightarrow n}^{K^{-}} v\right\rangle\left(1-X_{n}\right) n_{B} n_{K^{-}}-\left\langle\sigma_{n \rightarrow p}^{K^{-}} v\right\rangle X_{n} n_{B} n_{K^{-}}, \\
\frac{d n_{K_{L}^{0}}}{d t}=n_{N} \frac{\operatorname{Br}_{N \rightarrow K_{L}^{0}}}{\tau_{N}}-\Gamma_{\text {decay }}^{K_{L}^{0}} n_{K_{L}^{0}}-\left\langle\sigma_{p \rightarrow n}^{K_{L}^{0}} v\right\rangle\left(1-X_{n}\right) n_{B} n_{K_{L}^{0}}-\left\langle\sigma_{n \rightarrow p}^{K_{L}^{0}} v\right\rangle X_{n} n_{B} n_{K_{L}^{0}} .
\end{array}\right.
$$

Here the quantities

$$
\begin{aligned}
& \left(\frac{d X_{n}}{d t}\right)_{\pi}=\left(1-X_{n}\right) n_{\pi^{-}}\left\langle\sigma_{p \rightarrow n}^{\pi^{-}} v\right\rangle-X_{n} n_{\pi^{+}}\left\langle\sigma_{n \rightarrow p}^{\pi^{+}} v\right\rangle \\
& \quad \text { and } \\
& \left(\frac{d X_{n}}{d t}\right)_{K}=\left(1-X_{n}\right) n_{K}\left\langle\sigma_{p \rightarrow n}^{K} v\right\rangle-X_{n} n_{K}\left\langle\sigma_{n \rightarrow p}^{K} v\right\rangle
\end{aligned}
$$

are the rates of change of $X_{n}$ due to different mesons $\left(K=K^{-} / K_{L}^{0}\right) ; n_{B}$ is the baryon number density $n_{B}=\eta_{B} n_{\gamma}$. In equations for the number density of mesons $n_{h}$, the first term comes from HNLs, the second is due to decays of mesons, and the last term is due to $p \leftrightarrow n$ conversion. The time-temperature relation and the scale factor dynamics are provided by the solution of Eq. (B7), and the HNL number density may be obtained using Eq. (B5).

During times $t_{\mathrm{eq}} \simeq\left(\Gamma_{\text {decay }}^{h}\right)^{-1} \sim 10^{-8} \mathrm{~s}$, which are small in comparison to any other time scale in the system, the solution for $n_{h}$ reaches the dynamical equilibrium: 


$$
\begin{gathered}
n_{\pi^{-}}=\frac{n_{N} \cdot \mathrm{Br}_{N \rightarrow \pi^{-}}}{\tau_{N}\left(\Gamma_{\text {decay }}^{\pi^{-}}+\left\langle\sigma_{p \rightarrow n}^{\pi^{-}} v\right\rangle\left(1-X_{n}\right) n_{B}\right)}, \quad n_{\pi^{+}}=\frac{n_{N} \cdot \mathrm{Br}_{N \rightarrow \pi^{+}}}{\tau_{N}\left(\Gamma_{\text {decay }}^{\pi^{+}}+\left\langle\sigma_{n \rightarrow p}^{\pi^{+}} v\right\rangle\left(1-X_{n}\right) n_{B}\right)}, \\
n_{K}=\frac{n_{N} \cdot \mathrm{Br}_{N \rightarrow K}}{\tau_{N}\left(\Gamma_{\text {decay }}^{K}+\left\langle\sigma_{p \rightarrow n}^{K} v\right\rangle\left(1-X_{n}\right) n_{B}+\left\langle\sigma_{n \rightarrow p}^{K} v\right\rangle X_{n} n_{B}\right)},
\end{gathered}
$$

where $K=K^{-} / K_{L}^{0}$.

Therefore, we solve a single equation:

$$
\frac{X_{n}}{d t}=\left(\frac{d X_{n}}{d t}\right)_{\mathrm{SM}}+\left(\frac{d X_{n}}{d t}\right)_{\pi}+\left(\frac{d X_{n}}{d t}\right)_{K^{-}}+\left(\frac{d X_{n}}{d t}\right)_{K_{L}^{0}},
$$

where we use the meson number densities given by Eqs. (D12) and (D13) in the meson-driven conversion rates (D11). The results are shown in Fig. 7. Our main result is the right panel of Fig. 7, which shows that the value $T_{0}^{\mathrm{min}} \simeq 1.50 \mathrm{MeV}$ and that its variation as a function of the HNL mass is within $\pm 1 \%$.

With the help of Eqs. (D2) and (D5), we obtain the value of the neutron abundance driven solely by a given meson $h$. As long as $T \gtrsim T_{0}$ [see Eq. (8) and the left panel of Fig. 7], the weak interaction processes may be completely neglected, and the resulting $X_{n}$ are given by

$$
X_{n}^{\pi^{ \pm}}=\frac{\left\langle\sigma_{p \rightarrow n}^{\pi^{-}} v\right\rangle \cdot n_{\pi^{-}}}{\left\langle\sigma_{p \rightarrow n}^{\pi^{-}} v\right\rangle \cdot n_{\pi^{-}}+\left\langle\sigma_{n \rightarrow p}^{\pi^{+}} v\right\rangle \cdot n_{\pi^{+}}} \approx \frac{0.9 F_{c}^{\pi}(T)}{1+0.9 F_{c}^{\pi}(T)}, \quad X_{n}^{K^{-}} \approx \frac{2.46 F_{c}^{K}}{2.46 F_{c}^{K}+1}, \quad X_{n}^{K_{L}^{0}} \approx 0.32 .
$$

The values of $X_{n}^{\pi^{-} / K^{-}}$grow with the decrease of the temperature due to the growth of the Coulomb factor $F_{c}$, which enhances the rate of the $p \rightarrow n$ process.

The quantities (D15) provide us with the qualitative estimate of the value of $X_{n}$ in the presence of different mesons (Fig. 7). Below the kaon production threshold, $X_{n}^{h}=X_{n}^{\pi^{ \pm}}$. At larger masses, in order to find $X_{n}^{h}$ we need to set the whole right-hand side of Eq. (D10) to zero. Below the $K_{L}^{0}$ production threshold (which occurs at $m_{N}=m_{\phi}$ ), the value of $X_{n}^{h}$ grows, since charged kaons push $X_{n}$ to higher values than $X_{n}^{\pi^{-}}$. Above the neutral kaon production threshold, the ratio $\mathrm{Br}_{N \rightarrow K^{-}} / \mathrm{Br}_{N \rightarrow \pi^{-}}$increases (Fig. 6) and $X_{n}^{h}$ grows further. However, kaons $K_{L}^{0}$, which are present in small amounts, somewhat diminish this growth.

The value of $X_{n}^{h}\left(m_{N}\right)$ provides us with the mass dependence of $T_{0}^{\min }\left(m_{N}\right)$, which is the smallest temperature allowed by observations (cf. Fig. 1). We show it in Fig. 7 (right panel).

\section{SBBN evolution at $T<T_{0}^{\min }$}

If HNLs disappear from the plasma before neutrinos freeze out, the evolution of the neutron abundance and
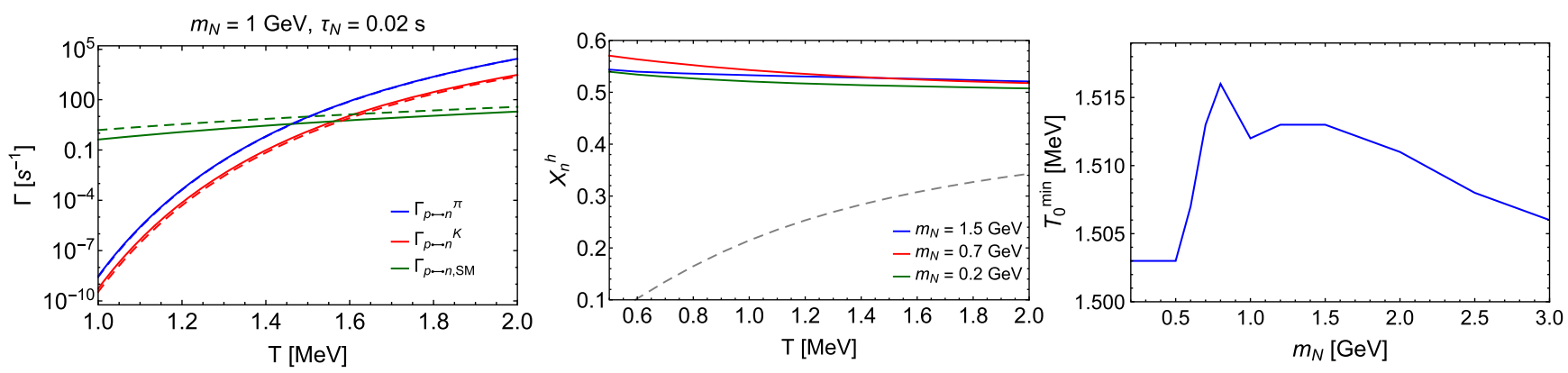

FIG. 7. Left panel: behavior of the $p \rightarrow n$ (solid lines) and $n \rightarrow p$ (dashed lines) conversion rates in the case of pion- and kaon-driven conversions and SBBN. We consider HNLs mixing with $e$ flavor, mass $m_{N}=1 \mathrm{GeV}$, and lifetime $\tau_{N}=0.02 \mathrm{~s}$ as an example. Middle panel: temperature dependence of the neutron abundance $X_{n}$ assuming that its evolution is completely dominated by the meson-driven $p \leftrightarrow n$ conversions. We consider HNLs mixing with $e$ flavor and different masses: $m_{N}=200 \mathrm{MeV}$ (only pions are present), $m_{N}=$ $700 \mathrm{MeV}$ (pions and charged kaons are present), and $m_{N}=1.5 \mathrm{GeV}$ (pions, and charged and neutral kaons are present). The dashed gray line denotes the value of the neutron abundance at equilibrium in SBBN. Right panel: HNL mass dependence of the temperature $T_{0}^{\min }$. 
subsequent nuclear reactions proceed exactly as in the SBBN case (albeit with a modified initial value of $X_{n}$ at $T=T_{0}^{\min }$ ).

Indeed, the onset of nuclear reactions is determined by the dynamical balance between reactions of deuterium synthesis and dissociation. This balance depends on the value of $\eta_{B}$. The latter gets diluted by the factor $\zeta$ due to decays of HNLs; see Appendix B. However, we fix $\eta_{B}$ to be the same as that measured by cosmic microwave background observations by the beginning of nuclear reactions. This of course means that $\eta_{B}$ has been $\zeta^{-1}$ times higher before decays of HNLs, but no observables can probe the value of $\eta_{B}$ in this epoch.

Another ingredient that affects the dynamics of nuclear reactions is the time-temperature relation, traditionally encoded in the value of $N_{\text {eff }}$. If HNLs have $\tau_{N} \ll 0.02 \mathrm{~s}$, neutrinos are in equilibrium and therefore HNL decays do not change $N_{\text {eff }}$; see the detailed analysis in Ref. [62].

As a result, the evolution of primordial plasma below $T_{0}^{\mathrm{min}}$ is governed by the SBBN equations.

\section{APPENDIX E: DISSOCIATION OF LIGHT ELEMENTS BY MESONS AND DOMAIN OF VALIDITY OF OUR TREATMENT}

The analysis presented in this paper is valid as long as HNLs decay before neutrino decoupling times. This puts an upper limit on the HNL lifetime [Eq. (15)]. It is clear, however, that once the lifetime is long enough and HNLs (their decay products) can survive until the onset of nuclear reactions, our treatment needs to be changed.

Indeed, pions, if present in the plasma at temperatures $T \lesssim T_{\text {BBN }}$, dissociate light nuclei. The ${ }^{4} \mathrm{He}$ thresholdless dissociation processes are (see Ref. [33])

$$
\begin{aligned}
& \pi^{-}+{ }^{4} \mathrm{He} \rightarrow T+n, \quad \pi^{-}+{ }^{4} \mathrm{He} \rightarrow D+2 n, \\
& \pi^{-}+{ }^{4} \mathrm{He} \rightarrow p+3 n .
\end{aligned}
$$

To estimate the lifetimes at which the processes (E1) can be neglected, we compare the number density of mesons available for the dissociation with the number density of ${ }^{4} \mathrm{He}$ nuclei:

$$
n_{\mathrm{He} \mathrm{diss}}^{h}\left(T_{\mathrm{BBN}}\right) \ll n_{\mathrm{He}}\left(T_{\mathrm{BBN}}\right),
$$

cf. Eq. (7). Here, $n_{\text {He diss }}^{\pi}$ is defined via

$$
n_{\text {He diss }}^{\pi}\left(T_{\mathrm{BBN}}\right)=n_{N} \cdot \mathrm{Br}_{N \rightarrow \pi^{-}} \cdot P_{\mathrm{He} \mathrm{diss}}
$$

and $P_{{ }^{4} \mathrm{He}}$ diss is the probability for a single meson to dissociate ${ }^{4} \mathrm{He}$ nuclei before decaying:

$P_{\mathrm{He} \mathrm{diss}}=\frac{\left\langle\sigma_{\mathrm{He} \mathrm{diss}}^{\pi} v\right\rangle n_{\mathrm{He}}}{\Gamma_{\text {decay }}^{\pi}} \simeq 8.3 \times 10^{-2} \cdot \frac{4 \cdot n_{\mathrm{He}}}{n_{B}}\left(\frac{T}{1 \mathrm{MeV}}\right)^{3}$,

where we used the total cross section of the dissociation processes $(\mathrm{E} 1), \quad\left\langle\sigma_{\mathrm{He} \mathrm{diss}}^{\pi} v\right\rangle \simeq 6.5 \cdot F_{\mathrm{He} \pi^{-}} \mathrm{mb} \quad[\mathrm{a}$ factor $F_{\mathrm{He} \pi^{-}} \simeq 3.5$ accounts for the Coulomb attraction (D3)].

In our estimates we use $T_{\mathrm{BBN}}=84 \mathrm{keV}$, assuming that all free nucleons become bounded in ${ }^{4} \mathrm{He}$ nuclei at this temperature. We also do not take into account that after the dissociation of helium the abundance of lighter elements will also be increased significantly. Assuming $n_{\mathrm{He}} \simeq n_{B} / 4$ in Eq. (E2), and using Eqs. (E3) and (E4) with the HNL number density given by Eq. (B8), we arrive at the upper bound on HNL lifetimes for which our analysis is applicable: $\tau_{N} \lesssim 40$ s. Notice that the dependence on $\zeta$ is only logarithmic, and therefore its exact value and its mass dependence play no role. The analysis for $\tau_{N}>40 \mathrm{~s}$ should be performed separately; see, e.g., Ref. [45].
[1] S. Alekhin et al., A facility to search for hidden particles at the CERN SPS: The SHiP physics case, Rep. Prog. Phys. 79, 124201 (2016).

[2] L. Canetti, M. Drewes, and M. Shaposhnikov, Matter and antimatter in the Universe, New J. Phys. 14, 095012 (2012).

[3] A. Boyarsky, M. Drewes, T. Lasserre, S. Mertens, and O. Ruchayskiy, Sterile neutrino dark matter, Prog. Part. Nucl. Phys. 104, 1 (2019).

[4] T. Asaka, S. Blanchet, and M. Shaposhnikov, The nuMSM, dark matter and neutrino masses, Phys. Lett. B 631, 151 (2005).
[5] T. Asaka and M. Shaposhnikov, The nuMSM, dark matter and baryon asymmetry of the Universe, Phys. Lett. B 620 , 17 (2005).

[6] A. Boyarsky, O. Ruchayskiy, and M. Shaposhnikov, The role of sterile neutrinos in cosmology and astrophysics, Annu. Rev. Nucl. Part. Sci. 59, 191 (2009).

[7] I. Boiarska et al., Probing baryon asymmetry of the Universe at LHC and SHiP, arXiv:1902.04535.

[8] J. Beacham et al., Physics beyond colliders at CERN: beyond the Standard Model working group report, J. Phys. G 47, 010501 (2020). 
[9] N. Aghanim et al., Planck 2018 results. VI. Cosmological parameters, Astron. Astrophys. 641, A6 (2020).

[10] Y. I. Izotov, T.X. Thuan, and N. G. Guseva, A new determination of the primordial $\mathrm{He}$ abundance using the He i $\lambda 10830 \AA$ emission line: Cosmological implications, Mon. Not. R. Astron. Soc. 445, 778 (2014).

[11] E. Aver, K. A. Olive, and E. D. Skillman, The effects of He I $\lambda 10830$ on helium abundance determinations, J. Cosmol. Astropart. Phys. 07 (2015) 011.

[12] A. Peimbert, M. Peimbert, and V. Luridiana, The primordial helium abundance and the number of neutrino families, Rev. Mex. Astron. Astrofis. 52, 419 (2016).

[13] V. Fernández, E. Terlevich, A. I. Díaz, R. Terlevich, and F. F. Rosales-Ortega, Primordial helium abundance determination using sulphur as metallicity tracer, Mon. Not. R. Astron. Soc. 478, 5301 (2018).

[14] M. Valerdi, A. Peimbert, M. Peimbert, and A. Sixtos, Determination of the primordial helium abundance based on NGC 346, an $\mathrm{H}$ ii region of the small magellanic cloud, Astrophys. J. 876, 98 (2019).

[15] C. Pitrou, A. Coc, J.-P. Uzan, and E. Vangioni, Precision big bang nucleosynthesis with improved Helium-4 predictions, Phys. Rep. 754, 1 (2018).

[16] A. Arbey, AlterBBN: A program for calculating the BBN abundances of the elements in alternative cosmologies, Comput. Phys. Commun. 183, 1822 (2012).

[17] E. Lisi, S. Sarkar, and F. L. Villante, The big bang nucleosynthesis limit on $\mathrm{N}($ neutrino), Phys. Rev. D 59, 123520 (1999).

[18] L. Mendoza and C. J. Hogan, A java calculator of standard big bang nucleosynthesis, arXiv:astro-ph/9904334.

[19] O. Pisanti, A. Cirillo, S. Esposito, F. Iocco, G. Mangano, G. Miele, and P. D. Serpico, PArthENoPE: Public algorithm evaluating the nucleosynthesis of primordial elements, Comput. Phys. Commun. 178, 956 (2008).

[20] M. Pospelov and J. Pradler, Big bang nucleosynthesis as a probe of new physics, Annu. Rev. Nucl. Part. Sci. 60, 539 (2010).

[21] A. Dolgov, S. Hansen, G. Raffelt, and D. Semikoz, Cosmological and astrophysical bounds on a heavy sterile neutrino and the KARMEN anomaly, Nucl. Phys. B580, 331 (2000).

[22] A. D. Dolgov, S. H. Hansen, G. Raffelt, and D. V. Semikoz, Heavy sterile neutrinos: Bounds from big bang nucleosynthesis and SN1987A, Nucl. Phys. B590, 562 (2000).

[23] A. Dolgov and F. Villante, BBN bounds on active sterile neutrino mixing, Nucl. Phys. B679, 261 (2004).

[24] G. M. Fuller, C. T. Kishimoto, and A. Kusenko, Heavy sterile neutrinos, entropy and relativistic energy production, and the relic neutrino background, arXiv:1110.6479.

[25] O. Ruchayskiy and A. Ivashko, Restrictions on the lifetime of sterile neutrinos from primordial nucleosynthesis, J. Cosmol. Astropart. Phys. 10 (2012) 014.

[26] P. Hernandez, M. Kekic, and J. Lopez-Pavon, Lowscale seesaw models versus $N_{\text {eff }}$, Phys. Rev. D 89, 073009 (2014).

[27] P. Hernandez, M. Kekic, and J. Lopez-Pavon, $N_{\text {eff }}$ in lowscale seesaw models versus the lightest neutrino mass, Phys. Rev. D 90, 065033 (2014).
[28] A. C. Vincent, E. F. Martinez, P. Hernández, M. Lattanzi, and O. Mena, Revisiting cosmological bounds on sterile neutrinos, J. Cosmol. Astropart. Phys. 04 (2015) 006.

[29] G. B. Gelmini, P. Lu, and V. Takhistov, Cosmological dependence of non-resonantly produced sterile neutrinos, J. Cosmol. Astropart. Phys. 12 (2019) 047.

[30] D. Kirilova, BBN cosmological constraints on beyond Standard Model neutrino, Proc. Sci., CORFU2018 (2019) 048.

[31] G. B. Gelmini, M. Kawasaki, A. Kusenko, K. Murai, and V. Takhistov, Big bang nucleosynthesis constraints on sterile neutrino and lepton asymmetry of the Universe, J. Cosmol. Astropart. Phys. 09 (2020) 051.

[32] N. Sabti, A. Magalich, and A. Filimonova, An extended analysis of heavy neutral leptons during big bang nucleosynthesis, J. Cosmol. Astropart. Phys. 11 (2020) 056.

[33] M. Pospelov and J. Pradler, Metastable GeV-scale particles as a solution to the cosmological lithium problem, Phys. Rev. D 82, 103514 (2010).

[34] A. Fradette and M. Pospelov, BBN for the LHC: Constraints on lifetimes of the Higgs portal scalars, Phys. Rev. D 96, 075033 (2017).

[35] A. Fradette, M. Pospelov, J. Pradler, and A. Ritz, Cosmological beam dump: Constraints on dark scalars mixed with the Higgs boson, Phys. Rev. D 99, 075004 (2019).

[36] M. H. Reno and D. Seckel, Primordial nucleosynthesis: The effects of injecting hadrons, Phys. Rev. D 37, 3441 (1988).

[37] K. Kohri, Primordial nucleosynthesis and hadronic decay of a massive particle with a relatively short lifetime, Phys. Rev. D 64, 043515 (2001).

[38] M. Kawasaki, K. Kohri, and T. Moroi, Big-Bang nucleosynthesis and hadronic decay of long-lived massive particles, Phys. Rev. D 71, 083502 (2005).

[39] D. Gorbunov and M. Shaposhnikov, How to find neutral leptons of the $\nu$ MSM? J. High Energy Phys. 10 (2007) 015; Erratum, J. High Energy Phys. 11 (2013) 101.

[40] K. Bondarenko, A. Boyarsky, D. Gorbunov, and O. Ruchayskiy, Phenomenology of GeV-scale heavy neutral leptons, J. High Energy Phys. 11 (2018) 032.

[41] S. Dodelson and L. M. Widrow, Sterile-Neutrinos as Dark Matter, Phys. Rev. Lett. 72, 17 (1994).

[42] X.-D. Shi and G. M. Fuller, A New Dark Matter Candidate: Nonthermal Sterile Neutrinos, Phys. Rev. Lett. 82, 2832 (1999).

[43] M. Tanabashi et al., Review of particle physics, Phys. Rev. D 98, 030001 (2018).

[44] Y. I. Izotov, G. Stasinska, and N. G. Guseva, Primordial 4He abundance: A determination based on the largest sample of HII regions with a methodology tested on model HII regions, Astron. Astrophys. 558, A57 (2013).

[45] K. Bondarenko et al., An allowed window for heavy neutral leptons below the kaon mass, arXiv:2101.09255.

[46] V. Domcke, M. Drewes, M. Hufnagel, and M. Lucente, MeV-scale seesaw and leptogenesis, J. High Energy Phys. 01 (2021) 200.

[47] S. Eijima, M. Shaposhnikov, and I. Timiryasov, Parameter space of baryogenesis in the $\nu$ MSM, J. High Energy Phys. 07 (2019) 077.

[48] J. Klarić, M. Shaposhnikov, and I. Timiryasov, Uniting lowscale leptogeneses, arXiv:2008.13771. 
[49] V. Syvolap, Astrophysical and cosmological constraints on novel particles, Ph.D. thesis, Niels Bohr Institute, 2021.

[50] E. Cortina Gil et al., Search for heavy neutral lepton production in $K^{+}$decays to positrons, Phys. Lett. B 807, 135599 (2020).

[51] C. Ahdida et al., Sensitivity of the SHiP experiment to heavy neutral leptons, J. High Energy Phys. 04 (2019) 077.

[52] D. Gorbunov, I. Krasnov, Y. Kudenko, and S. Suvorov, Heavy neutral Leptons from kaon decays in the SHiP experiment, Phys. Lett. B 810, 135817 (2020).

[53] P. Ballett, T. Boschi, and S. Pascoli, Heavy neutral leptons from low-scale seesaws at the DUNE near detector, J. High Energy Phys. 20 (2020) 111.

[54] B. Abi et al., Deep underground neutrino experiment (DUNE), far detector technical design report, volume II DUNE physics, arXiv:2002.03005.

[55] P. Coloma, E. Fernández-Martínez, M. González-López, J. Hernández-García, and Z. Pavlovic, GeV-scale neutrinos: Interactions with mesons and DUNE sensitivity, Eur. Phys. J. C 81, 78 (2021).
[56] O. Ruchayskiy and A. Ivashko, Experimental bounds on sterile neutrino mixing angles, J. High Energy Phys. 06 (2012) 100 .

[57] R. K. Ellis et al., Physics briefing book: Input for the european strategy for particle physics update 2020, arXiv:1910.11775.

[58] Y. I. IzotovF. H. Chaffee, C. B. Foltz, R. F. Green, N. G. Guseva, and T.X. Thuan, Helium abundance in the most metal-deficient blue compact galaxies: I zW 18 and sbs 0335-052, Astrophys. J. 527, 757 (1999).

[59] K. A. Olive and E. D. Skillman, A realistic determination of the error on the primordial helium abundance: Steps toward non-parametric nebular helium abundances, Astrophys. J. 617, 29 (2004).

[60] M. Shaposhnikov, A possible symmetry of the nuMSM, Nucl. Phys. B763, 49 (2007).

[61] J. Kersten and A. Y. Smirnov, Right-handed neutrinos at CERN LHC and the mechanism of neutrino mass generation, Phys. Rev. D 76, 073005 (2007).

[62] A. Boyarsky, M. Ovchynnikov, N. Sabti, and V. Syvolap, When FIMPs decay into neutrinos: The $N_{\text {eff }}$ story, arXiv: 2103.09831. 